\title{
Dynamics of a Linear Beam with an Attached Local Nonlinear Energy Sink - NES
}

\author{
F. Georgiades and A.F. Vakakis (†)
}

Mechanics Division,

Faculty of Applied Mathematical and Physical Sciences, National Technical University of Athens, fgeorgia@mail.ntua.gr, vakakis@central.ntua.gr

$\uparrow$ Corresponding author,

P.O.Box 64042, GR-157 10 Zografos, Athens, Greece

Department of Mechanical and Industrial Engineering (adjunct), Department of Aerospace Engineering (adjunct), University of Illinois at Urbana-Champaign, avakakis@uiuc.edu

\begin{abstract}
We provide numerical evidence of passive and broadband targeted energy transfer from a linear flexible beam under shock excitation to a local essentially nonlinear lightweight attachment that acts, in essence, as nonlinear energy sink-NES. It is shown that the NES absorbs shock energy in a one-way, irreversible fashion and dissipates this energy locally, without_spreading_it back to the linear beam. Moreover, we show numerically that an appropriately designed and placed NES can passively absorb and locally dissipate a major portion of the shock energy of the beam, up to an optimal value of $87 \%$. The implementation of the NES concept to the shock isolation of practical engineering structures and to other applications is discussed.
\end{abstract}




\section{Introduction}

In previous works $[1,2]$ it was shown that essentially nonlinear oscillators attached to linear discrete structures can act as broadband passive absorbers of vibration energy. In particular, it was shown that transient resonance captures of the transient dynamics in neighborhoods of resonance manifolds of the phase space of a dynamical system [3,4] may initiate one-way, irreversible targeted energy transfer from a linear (main) subsystem to a local essentially nonlinear attachment, which acts, in essence, as nonlinear energy sink (NES) [5,6]. Moreover, in contract to the classical linear vibration absorber, the NES may passively absorb broadband energy over wide frequency ranges; this due to the essential stiffness nonlinearity of the NES, which enables resonance capture of the NES with any mode of the linear subsystem, irrespective of its frequency.

Up to now passive targeted energy transfer was studied only in discrete systems of coupled oscillators. This communication demonstrates both computationally and experimentally that the NES concept can be extended to flexible systems as well. Specifically, we show that an appropriately designed and placed essentially nonlinear local attachment may absorb and locally dissipate a significant portion of shock-induced vibration energy of a beam, a result that paves the way for the practical implementation of the NES concept to flexible systems.

\section{Dynamical System and Truncated Model}

The system considered in appears in Figure 1. It consists of an impulsively forced, simply supported, damped linear beam, with an attached essentially nonlinear, damped single-degree-offreedom oscillator. We will show that the nonlinear attachment can passively and irreversibly absorb a major portion of the vibration energy of the beam induced by the impulse, thus acting as NES. Moreover, the targeted energy transfer from the linear beam to the NES can be optimized by appropriate design and placement of the attachment.

(Figure 1)

Assuming that the beam dynamics is governed by linear Bernoulli theory, the equations of motion of the system are given by,

$$
\begin{gathered}
\mathrm{EI}_{\mathrm{xxxx}}(\mathrm{x}, \mathrm{t})+\varepsilon \lambda \mathrm{y}_{\mathrm{t}}(\mathrm{x}, \mathrm{t})+\mathrm{my}_{\mathrm{tt}}(\mathrm{x}, \mathrm{t})+\left\{\mathrm{C}[\mathrm{y}(\mathrm{d}, \mathrm{t})-\mathrm{v}(\mathrm{t})]^{3}+\varepsilon \lambda\left[\mathrm{y}_{\mathrm{t}}(\mathrm{d}, \mathrm{t})-\dot{\mathrm{v}}(\mathrm{t})\right]\right\} \delta(\mathrm{x}-\mathrm{d})=\mathrm{F}(\mathrm{t}) \delta(\mathrm{x}-\mathrm{a}) \\
\varepsilon \ddot{\mathrm{v}}(\mathrm{t})+\mathrm{C}[\mathrm{v}(\mathrm{t})-\mathrm{y}(\mathrm{d}, \mathrm{t})]^{3}+\varepsilon \lambda\left[\dot{\mathrm{v}}(\mathrm{t})-\mathrm{y}_{\mathrm{t}}(\mathrm{d}, \mathrm{t})\right]=0
\end{gathered}
$$

with zero initial conditions. Assuming that $0<\varepsilon<<1$, the system possesses light viscous damping, and the attachment is lightweight compared to the beam. This last assumption is important for the practical implementation of the NES concept. In addition, the attachment possesses essential cubic stiffness nonlinearity, which, together with viscous damping dissipation are prerequisites for the realization of targeted energy transfer in this system [1,2].

The transient response of system (1) is computed by projecting the dynamics of the partial differential equation in the complete and orthonormal base of the linear vibration modes of the simply supported beam with no nonlinear attachment,

$$
\phi_{\mathrm{r}}(\mathrm{x})=(2 / \mathrm{mL})^{1 / 2} \sin (\mathrm{r} \pi \mathrm{x} / \mathrm{L}), \quad \omega_{\mathrm{r}}=(\mathrm{r} \pi)^{2}\left(\mathrm{EI} / \mathrm{mL}^{4}\right)^{1 / 2}, \quad \mathrm{r}=1,2, \ldots
$$

where $\omega_{\mathrm{r}}$ is the natural frequency of the $r$-th mode. The base eigenfunctions satisfy the following modal equations and orthonormality conditions, 


$$
\phi_{\mathrm{r}}^{\prime \prime}(\mathrm{x})+\omega_{\mathrm{r}}^{2} \phi_{\mathrm{r}}(\mathrm{x})=0, \quad \int_{0}^{\mathrm{L}} \mathrm{m} \phi_{\mathrm{i}}(\mathrm{x}) \phi_{\mathrm{j}}(\mathrm{x}) \mathrm{dx}=\delta_{\mathrm{ij}}, \quad \mathrm{i}, \mathrm{j}, \mathrm{r}=1,2, \ldots
$$

where $\delta_{\mathrm{ij}}$ is Kronecker's symbol.

To project the dynamics of (1) in the infinite-dimensional orthonormal basis (2) we express the transverse displacement field $\mathrm{y}(\mathrm{x}, \mathrm{t})$ in the series form,

$$
y(x, t)=\sum_{r=1}^{\infty} a_{r}(t) \phi_{r}(x)
$$

which upon substitution in (1) and use of the above orthonormality relations yields the following infinite set of coupled oscillators with cubic stiffness nonlinearities:

$$
\begin{gathered}
\ddot{a}_{\mathrm{p}}(\mathrm{t})+\omega_{\mathrm{p}}^{2} \mathrm{a}_{\mathrm{p}}(\mathrm{t})+(\varepsilon \lambda / \mathrm{m}) \dot{\mathrm{a}}_{\mathrm{p}}(\mathrm{t})+ \\
\left\{\mathrm{C}\left[\sum_{\mathrm{r}=1}^{\infty} \mathrm{a}_{\mathrm{r}}(\mathrm{t}) \phi_{\mathrm{r}}(\mathrm{d})-\mathrm{v}(\mathrm{t})\right]^{3}+\varepsilon \lambda\left[\sum_{\mathrm{r}=1}^{\infty} \dot{\mathrm{a}}_{\mathrm{r}}(\mathrm{t}) \phi_{\mathrm{r}}(\mathrm{d})-\dot{\mathrm{v}}(\mathrm{t})\right]\right\} \phi_{\mathrm{p}}(\mathrm{d})=\mathrm{F}(\mathrm{t}) \phi_{\mathrm{p}}(\mathrm{a}) \\
\varepsilon \ddot{\mathrm{v}}(\mathrm{t})+\mathrm{C}\left[\mathrm{v}(\mathrm{t})-\sum_{\mathrm{r}=1}^{\infty} \mathrm{a}_{\mathrm{r}}(\mathrm{t}) \phi_{\mathrm{r}}(\mathrm{d})\right]^{3}+\varepsilon \lambda\left[\dot{\mathrm{v}}(\mathrm{t})-\sum_{\mathrm{r}=1}^{\infty} \dot{\mathrm{a}}_{\mathrm{r}}(\mathrm{t}) \phi_{\mathrm{r}}(\mathrm{d})\right]=0
\end{gathered}
$$

where $p=1,2, \ldots$ We note that the essential nonlinearity couples all modes through the infinite summation terms, whereas, the linear part of the system uncouples completely.

To numerically solve the set (4) in order to compute the transient dynamics of the system of Figure 1, it is necessary to truncate the expansion (3) to a finite number of modes, e.g., to perform an approximate projection of the dynamics to a finite-dimensional basis of orthonormal eigenfunctions. A numerical convergence analysis was performed, and it was found that $\mathrm{N}=5$ modes are sufficient for accurately computing the transient dynamics. Examples of two such convergence results are depicted in Figures $2 \mathrm{a}$ and $2 \mathrm{~b}$, where the portion of input energy dissipated by the damper of the NES at $\mathrm{t}=150$ is plotted as function of the nonlinear coefficient $\mathrm{C}$ and the position $d$ of the NES. For these simulations the impulsive force was selected as the half sine pulse,

$$
\mathrm{F}(\mathrm{t})=\left\{\begin{array}{cc}
\mathrm{A} \sin (2 \pi \mathrm{t} / \mathrm{T}), & 0 \leq \mathrm{t} \leq \mathrm{T} / 2 \\
0, & \mathrm{t}>\mathrm{T} / 2
\end{array}\right.
$$

with $A=10.0, T=0.4 / \pi$, and the system parameters were assigned the values,

$$
\mathrm{EI}=1.0, \mathrm{~m}=1.0, \mathrm{~L}=1.0, \varepsilon=0.1, \mathrm{a}=0.3, \lambda=0.5
$$

and $\mathrm{d}=0.65$ for Figure $2 \mathrm{a}, \mathrm{C}=1.322 \times 10^{3}$ for Figure $2 \mathrm{~b}$. The portion of input energy dissipated at the damper of the NES at time $t$ is computed by the expression,

$$
\eta(t) \equiv \frac{E_{N E S}(t)}{E_{\text {in }}}=\frac{\int_{0}^{t} \varepsilon \lambda\left[\dot{v}(t)-\sum_{r=1}^{N} \dot{a}_{r}(t) \phi_{r}(d)\right]^{2} d \tau}{\int_{0}^{T} F(t) \sum_{r=1}^{N} \dot{a}_{r}(t) \phi_{r}(a) d t}
$$

where $\mathrm{N}$ is the number of modes retained in the expansion (3). It is expected that for shock excitation of the damped system (1), as time increases the energy ratio (7) reaches an asymptotic limit $\eta_{t>1}$ representing the total portion of input shock energy of the beam that is passively absorbed and locally dissipated by the NES. In the numerical simulations of Figures $2 \mathrm{a}, \mathrm{b}$ the limiting ratio $\eta_{t>>1}$ was computed by computing the dynamics up to $t=150$, since by that time 
instant the transient dynamics was nearly damped, and the asymptotic limit of energy dissipation $\eta_{\mathrm{t}>>1}$ was approximately reached.

Studying the plots of Figures $2 \mathrm{a}, \mathrm{b}$ we note convergence of the results for $\mathrm{N}=5$ modes, justifying the mode truncation implemented in the following results. Considering the dependence of the portion of energy dissipated at the NES on the nonlinear coefficient (Fig.2a), we note that for $\mathrm{C}$ of $\mathrm{O}\left(10^{3}\right)$ it is significant, reaching as high as 0.87 . Considering the dependence of the portion of energy dissipated at the NES on its position (Fig.2b), we note two regions of high values (of the order of 0.85 ) when the NES is placed between the boundaries and the center of the beam, whereas significantly lower values are realized when the NES is placed near the center of the beam (where the second and fourth modes possess a node), or near the boundaries of the beam where all modes possess zero displacements. These results indicate that an appropriately designed and placed NES can passively absorb and dissipate a major portion of the energy induced to the beam by the external shock; moreover, this passive energy absorption is broadband and irreversible (e.g., once it is absorbed by the NES is does not 'spread back' to the beam), as verified by the significant levels of energy that is eventually dissipated by the damper of the NES.

In the following section we present the results of an optimization study of the NES with the goal of maximizing the portion of the input energy that is eventually absorbed and dissipated by the NES, and discuss the implementation of the NES concept to shock isolation of flexible systems.

(Figure 2)

\section{Optimization of Passive Energy Transfer and Dissipation at the NES}

The following simulations were performed for shock excitation (5) with $A=10.0, T=0.4 / \pi$, and system parameters, $\mathrm{EI}=1.0, \mathrm{~m}=1.0, \mathrm{~L}=1.0, \varepsilon=0.1, \mathrm{a}=0.3, \lambda=0.5 . \mathrm{In}$ addition, motivated by the convergence study we truncated the discretized set of equations (4) by retaining only the five leading modes of the linear beam. Keeping the (light) mass of the NES constant we computed the approximate asymptotic limit $\eta_{t>>1}$ reached by the NES energy measure $\eta$ defined in (7). In Figure 3 the asymptotic energy limit $\eta_{t>>1}$ is depicted as function of the nonlinearity coefficient $\mathrm{C}$ and the position $\mathrm{d}$ of the NES (viewed in context, the plots of Figure 2 can be regarded as being two 'slices' of the three dimensional plot of Figure 3a).

(Figure 3)

There are two regions of effective performance of the NES in the (C,d) plane, with optimal NES energy dissipation reaching the value of 0.833 realized for $C=1.320 \times 10^{3}$ and $\mathrm{d}=0.348$. Moreover, for $\mathrm{C}$ of $\mathrm{O}\left(10^{3}\right)$ the effectiveness of the NES appears to be robust in variations of $\mathrm{C}$; this is indicated by the two distinct 'strips' of high values of energy dissipation in the plots of Figure 3. At the same time, the plots of Figure 3 reveal a strong dependence of the portion of energy dissipated by the NES on its placement position d. This should be expected, given that, by placing the NES close to the center or the boundaries of the beam prevents strong absorption and dissipation of shock energy from beam modes possessing nodes near the position of the NES. However, a general conclusion drawn from the plots of Figures 3 is that $a$ lightweight, essentially nonlinear NES can be designed and appropriately placed to passively absorb a major portion (of the order of 80\%) of shock energy induced in the beam. This result is novel, as the described energy absorption is broadband (as it involves shock energy and multi- 
modal beam resepose), realized over wide frequency ranges; as such, it contrasts clearly to the action of classical linear absorbers where energy absorption is narrowband.

(Figure 4)

To demonstrate the significant reduction of the level of beam vibrations caused by the action of the NES, in Figure 4 we depict the transient responses of the NES and the point of attachment of the beam for the system with parameters $C=1.320 \times 10^{3}$ and $d=0.65$. For comparison purposes we also show the response of the same point of the beam with no NES attached. Note the drastic reduction of the envelope of oscillation of the point of the beam caused by the rapid absorption and dissipation of energy by the NES. The multi-frequency content clearly evidenced in the NES transient response (high frequencies at early time with a transition to lower frequencies as time increases) indicates absorption of energy from different structural modes of the beam, and demonstrates clearly the capacity of the NES to absorb and dissipated broadband energy from the beam.

The dynamical mechanisms governing passive targeted energy transfer from linear to nonlinear oscillators are discussed in previous works [5,6]. Two of these mechanisms rely on resonance capture of the damped dynamics on either fundamental or subharmonic resonant manifolds in the phase space of the dynamical system; viewed from an alternative perspective, irreversible targeted energy transfer from the linear oscillator to the nonlinear attachment takes place when the dynamics is restricted to a damped nonlinear normal mode invariant manifold, whose mode shape becomes strongly localized to the nonlinear attachment as the energy deceases due to damping dissipation. A third mechanism for passive targeted energy transfer relies on nonlinear beat phenomena to initiate (but not cause) strong energy pumping; these beats act as 'bridging orbits' (or 'catalysts') for facilitating energy transfer by activating either one of the previous fundamental or subharmonic resonance capture mechanisms. It is interesting to note that these phenomena occur in spite of the lightness of the NES compared to the linear oscillator and the complete absence of any active (energy source) element in the system. These dynamical mechanisms are expected to be present in the numerical results presented in this note, and to govern passive targeted energy transfer from linear flexible systems to attached NESs.

\section{Concluding Remarks}

The considered nonlinear attachment holds promise as an efficient, robust, lightweight and modular passive absorbing device for eliminating undesired shock-induced broadband disturbances in small- or large-scale flexible structures. Viewed in that context, the NES can be viewed as a passive, broadband boundary controller, and as such can find application in diverse problems in engineering and physics, including shock isolation of machines and structures, seismic mitigation, packaging, and instability suppression (such as limit cycle aeroelastic oscillations or flutter). 


\section{References}

1. VAKAKIS A.F., MANEVITCH L.I., GENDELMAN O., BERGMAN L. 2003, Journal of Sound and Vibration, 264, 559-577, Dynamics of linear discrete systems connected to local essentially nonlinear attachments.

2. PANAGOPOULOS, P.N., VAKAKIS, A.F., TSAKIRTZIS, S. 2004, International Journal of Solids and Sructures, Transient resonant interactions of linear chains with essentially nonlinear end attachments leading to passive energy pumping.

3. QUINN, D. AND RAND, R.H. 1995, Nonlinear Dynamics 8, 1-20, The Dynamics of Resonance Capture.

4. QUINN, D. 1997, Nonlinear Dynamics, 14, 309-333, Resonance Capture in a Three Degree of Freedom Mechanical System.

5. LEE Y.S., KERSCHEN G., VAKAKIS A.F., PANAGOPOULOS P.N., BERGMAN L.A., McFARLAND D.M. 2005, Physica D, Complicated Dynamics of a Linear Oscillator with a Light, Essentially Nonlinear Attachment (in press).

6. KERSCHEN G., LEE Y.S., VAKAKIS A.F., McFARLAND D.M., BERGMAN L.A., 2005, SIAM Journal on Applied Mathematics, Irreversible Passive Energy Transfer in Coupled Oscillators with Essential Nonlinearity (submitted). 


\section{Figure Captions}

1. Linear beam with local, essentially nonlinear NES.

2. Convergence study of the portion of input energy absorbed and dissipated by the NES, for a truncated system with $\mathrm{N}=1,2$ and 5 modes, as function of (a) the NES stiffness $\mathrm{C}$, (b) the NES position d.

3. Dependence of the portion of input energy absorbed and dissipated by the NES as function of the NES stiffness $\mathrm{C}$ and position d: (a) full three-dimensional plot, (b) contour projection in the $(\mathrm{C}, \mathrm{d})$ plane.

4. Transient responses of, (a) the NES, (b) the point of attachment of the beam, for $\mathrm{C}=1.322 \times 10^{3}$ and $\mathrm{d}=0.65$. 
Figures

Figure 1

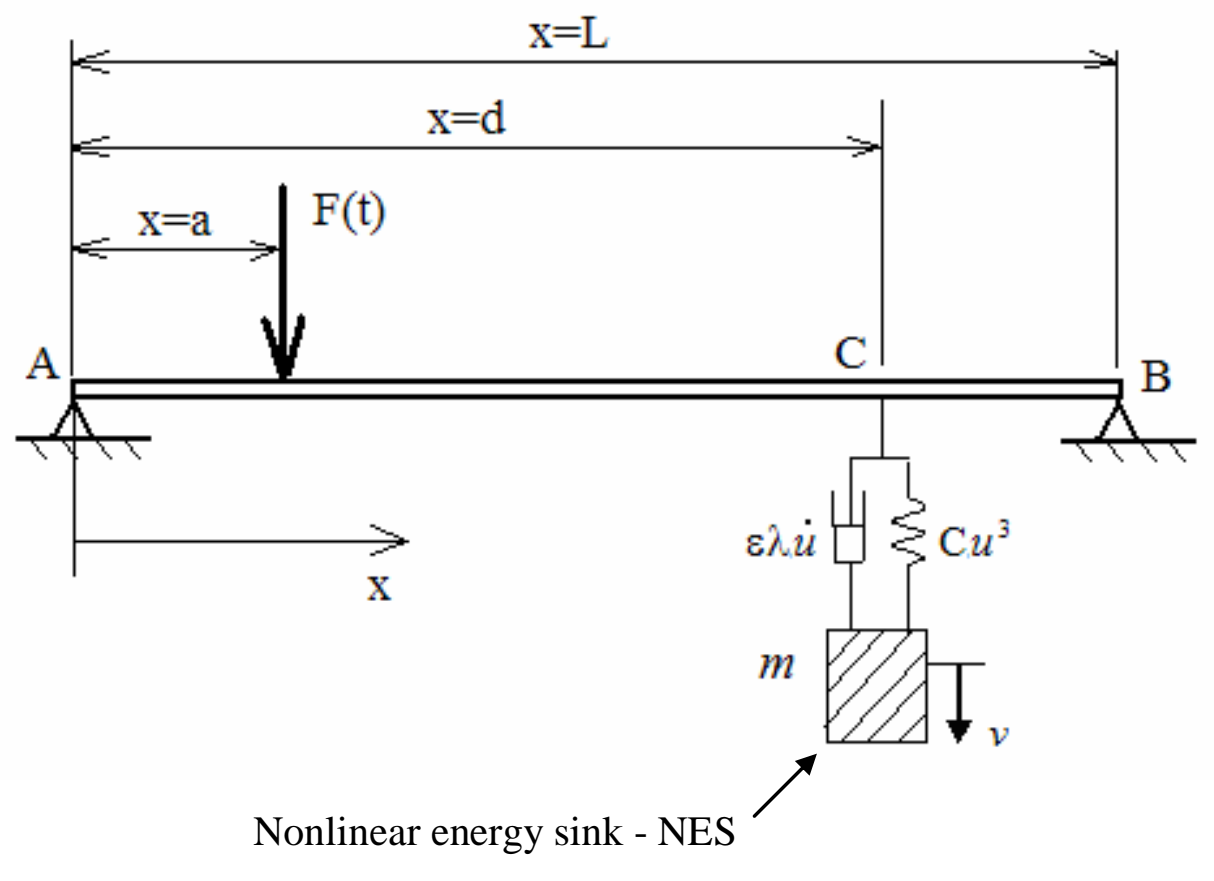




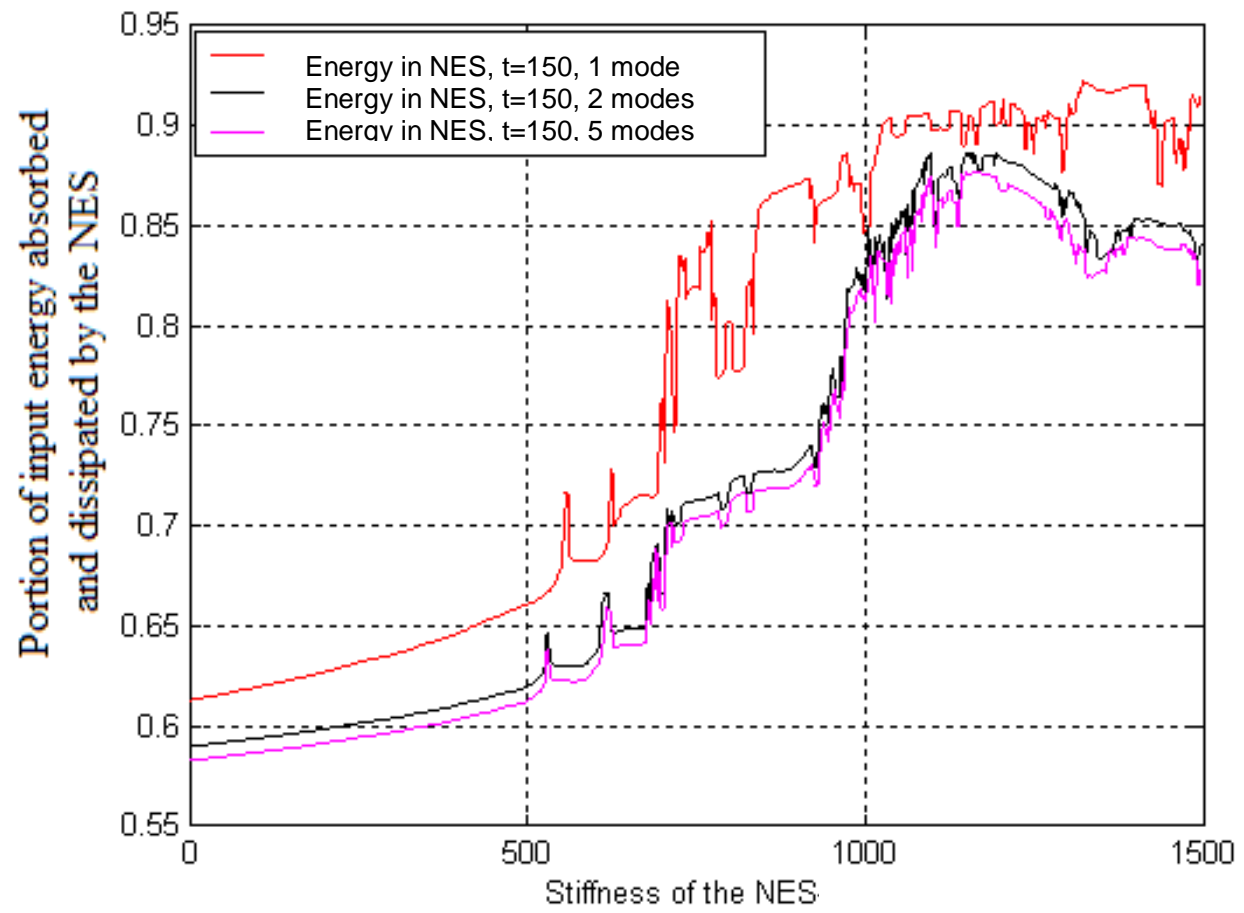

(a)

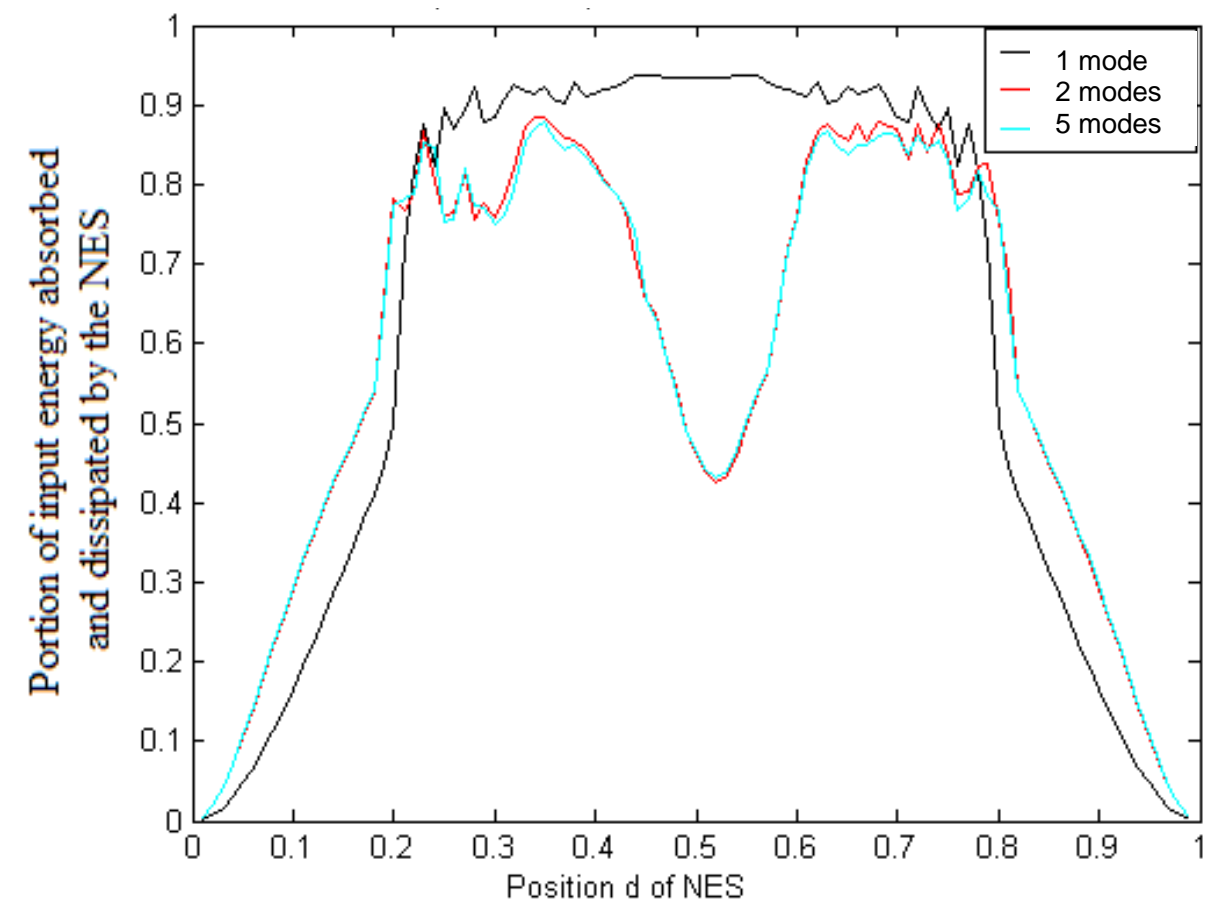

(b)

Figure 2 

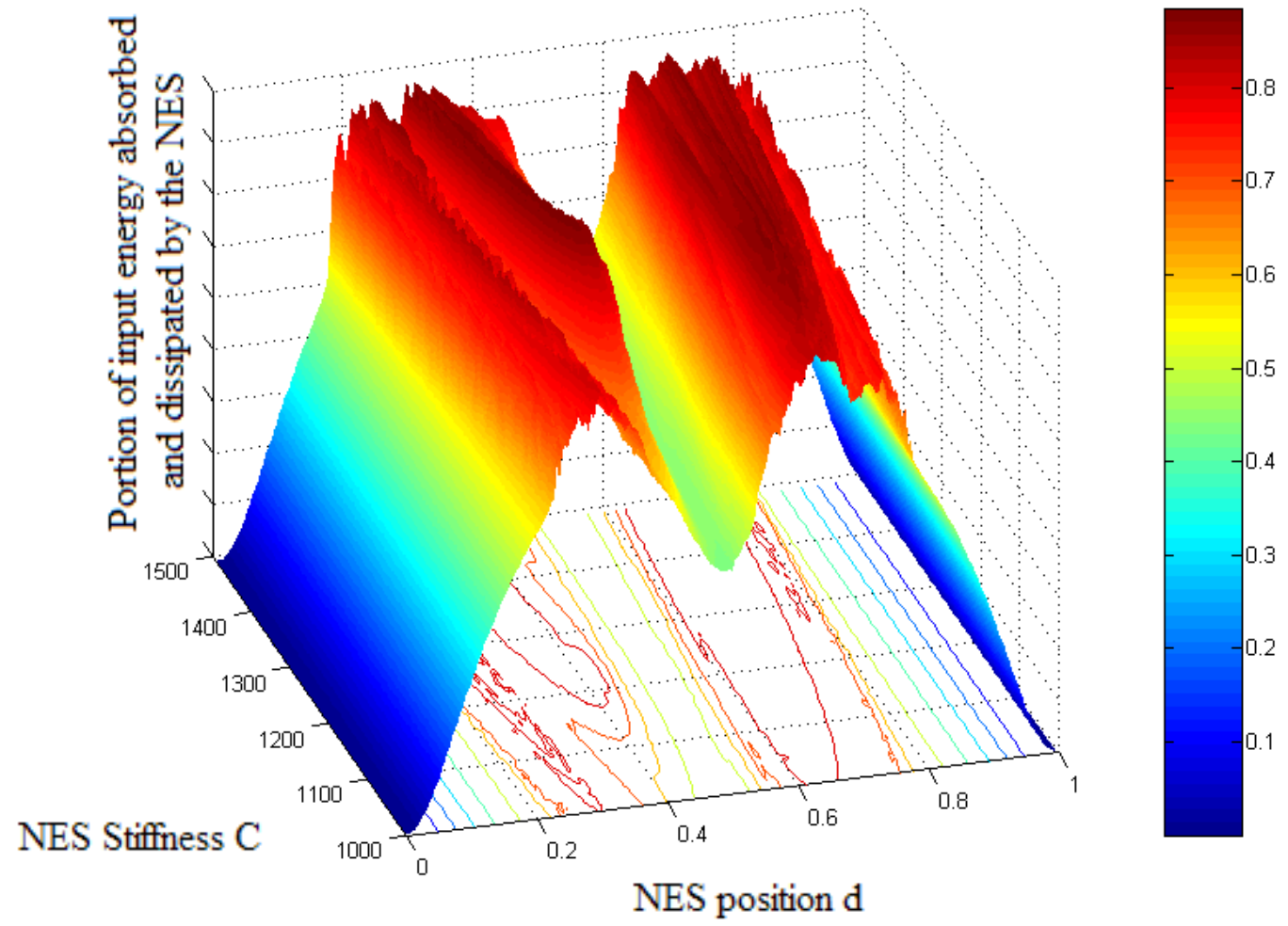

(a)

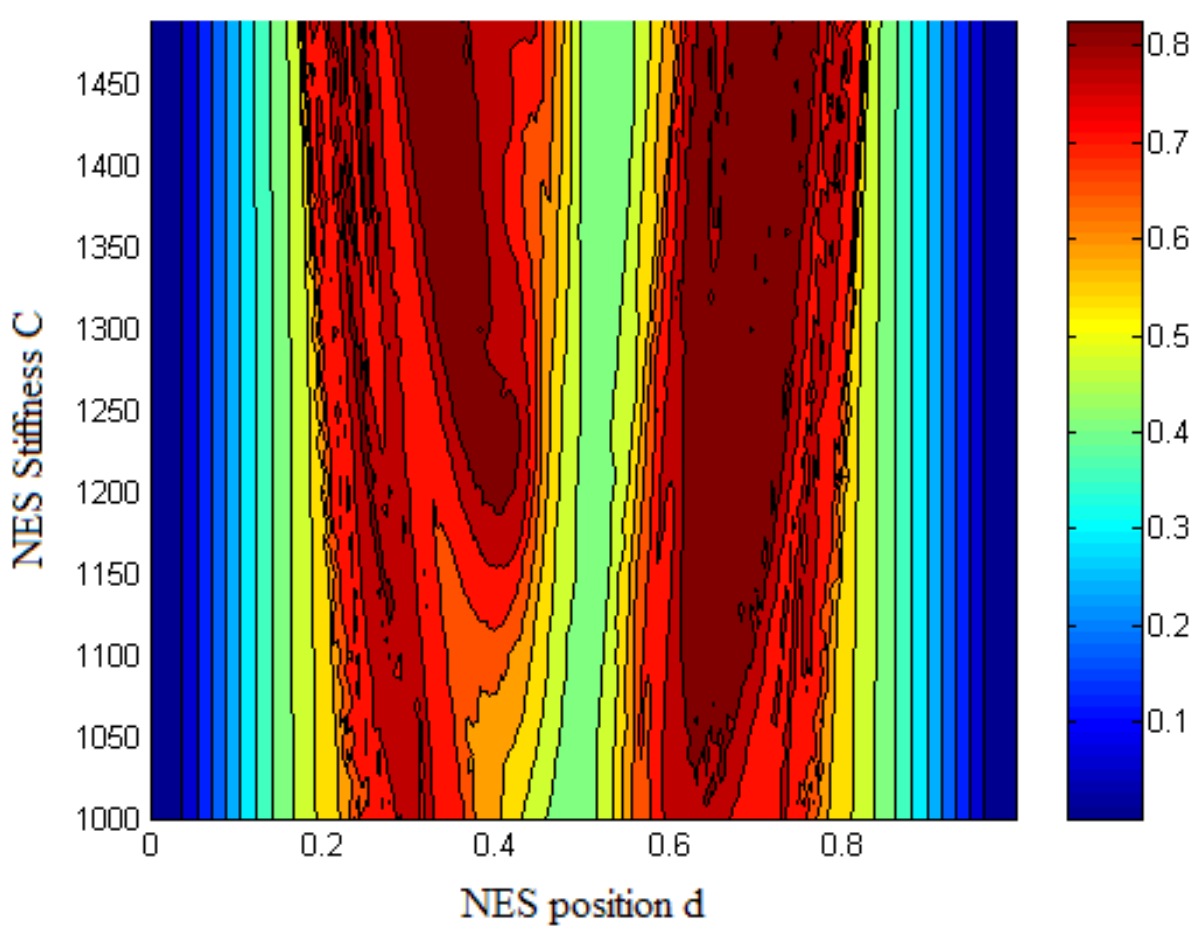

(b)

Figure 3 


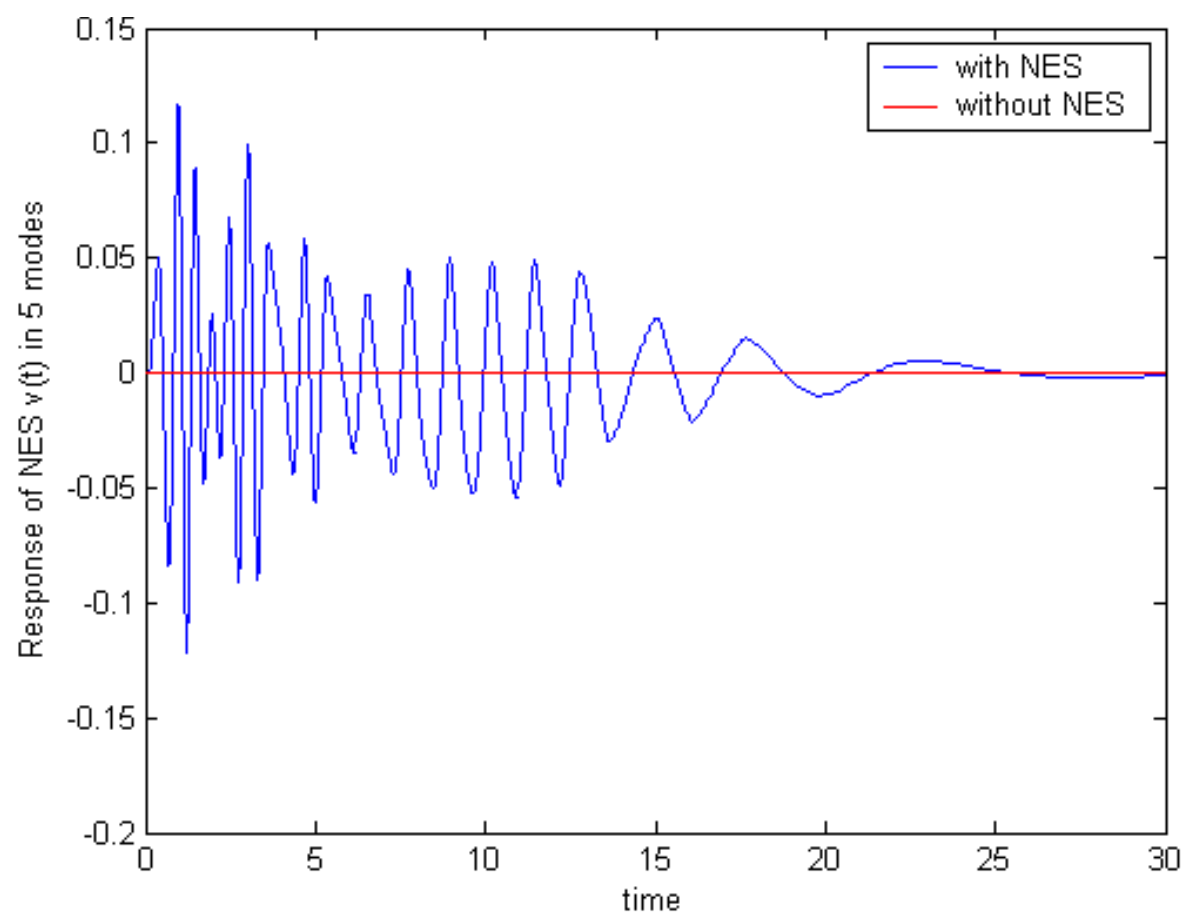

(a)

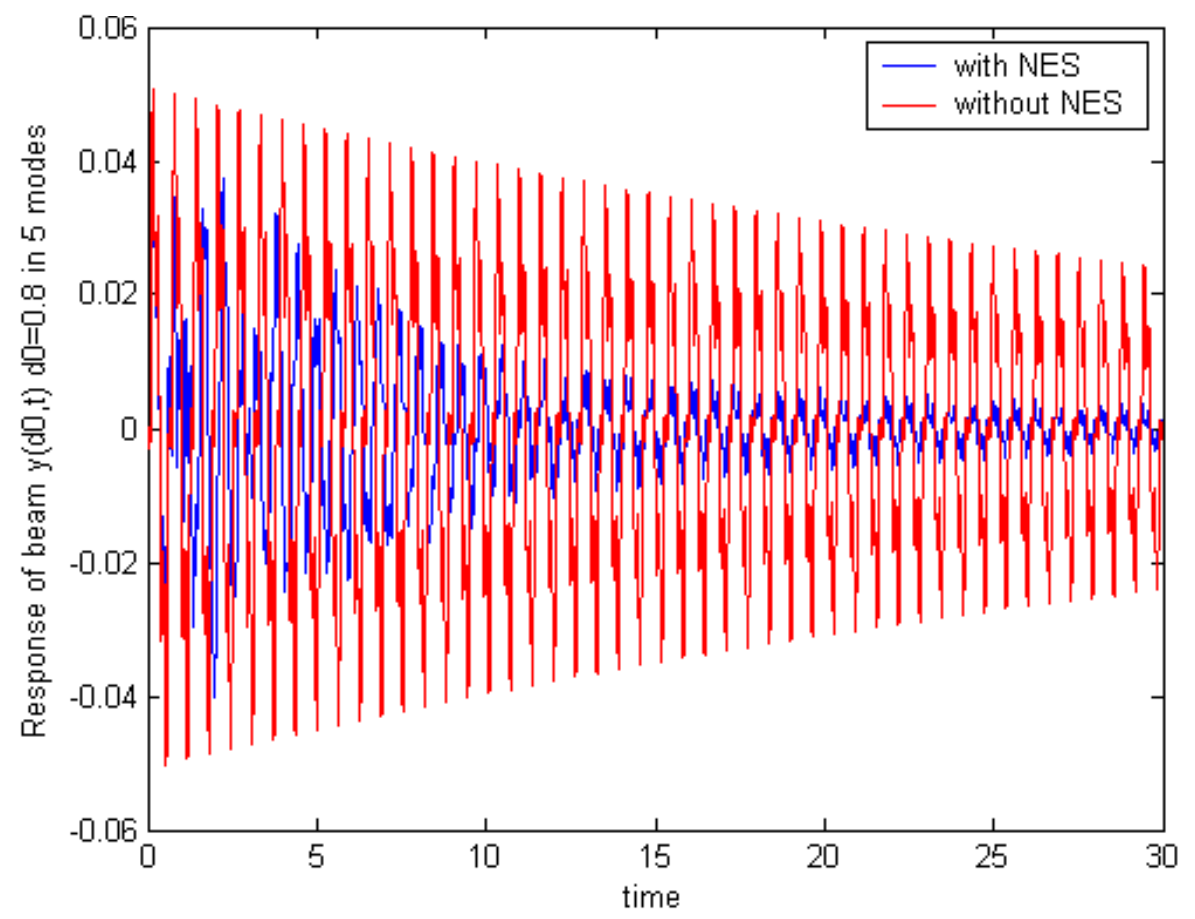

(b)

Figure 4 